704

\section{Verbal fluency and semantic memory in schizophrenia}

SIR: Allen et al (BJP, December 1993, 163, 769$775)$ reported that with a verbal fluency task repeated at different intervals, schizophrenic patients tended to produce fewer items than control subjects. The sets of items produced on different occasions by the same patient tended to be varied. By comparing items produced on different occasions, it was concluded that patients may have a mental lexicon similar to that of normal controls, and that the main deficit in the verbal fluency task lies in retrieval.

Studies of memory deficits could be divided into studies of recall and studies of recognition. While recall requires an active search, recognition reflects more directly the contents of the memory store. A verbal fluency test could be considered as a recall test of the long-term semantic store; another way to study this store is by a recognition test. Recognition for semantic categories could be tested with a paradigm where subjects are asked to decide whether a presented exemplar (e.g. 'chair') belongs to a particular category (e.g. 'furniture'). This paradigm has been used in a study of schizophrenic patients (Chen et al, 1994), and one of the findings was that patients responded normally most of the time to items within the category (i.e. making correct decisions, with faster response to more typical items). This concurs with the findings of Allen $e t a l$ in suggesting that the mental lexicon in schizophrenic patients is probably not grossly degraded, even in the presence of severe poverty of speech or incoherence. This is an important negative finding.

A similar profile of impaired retrieval in the presence of intact semantic store has also been described in patients with Parkinson's disease, Huntington's chorea (Randolph et al, 1993), and frontal lobe lesions (Shimamura et al, 1991), suggesting that in schizophrenia a similar set of neural substrates (basal ganglia, prefrontal cortex, among others) is involved in the impairment of verbal fluency.

What is important to rule out, however, is the effect of medication. Unfortunately, the analysis of this appears not to have been presented by Allen $e t$ al. At least theoretically, antipsychotic medication may affect basal ganglia function and lead to a deficit of verbal fluency unrelated to the schizophrenic illness itself.

Chrn, E.Y.H., Wil.kins, A.J. \& McKfnNa, P.J. (1994) Semantic memory is both impaired and anomalous in schizophrenia. Psichological Medicine (in press).
RaNDOLPH, C., Braun, A.R., Goldberg, T.E., el al (1993) Semantic fluency in Alzheimer's, Parkinson's, and Huntington's disease: dissociation of storage and retrieval failures. Neuropsychology, 7, 82-88.

Shimamura, A.P. Janowsky, J.S. \& Souire, L.R. (1991) What is the role of frontal lobe damage in memory disorders? In Frontal Lobe Function and Dysfunction (eds H.S. Levin, H.M. Eisenberg \& A.L. Benton), pp. 173-198. Oxford: Oxford University Press.

ERIC Y.H. CHEN

Department of Psychiatry

University of Hong Kong

Severity and the stressor criterion in post-traumatic stress disorder

SIR: Lillywhite \& Neal (BJP, December 1993, 163, 837 ) raise some questionable criticisms of the case report of Spector \& Huthwaite (BJP, July 1993, 163, 106-108).

They complain of insufficient evidence that the patient's experience of a road traffic accident met the requirements of criterion $A$ "of being outside the range of normal human experience", and thus declare themselves "not convinced" by the diagnosis of post-traumatic stress disorder (PTSD).

It is unfair to criticise omissions from a brief clinical report, where editorial requirements do not allow the inclusion of many details. There are numerous unchallenged reports in the literature of PTSD following road traffic accidents-March (1993) lists them as a "characteristic" (PTSD stressor - though this common cause of severe and life-threatening stress (Epstein, 1993) has been grossly underinvestigated. Feinstein \& Dolan (1991) and others found that the perceived severity of an accident does not necessarily relate to the onset of PTSD, but more than half of those injured in car accidents perceive the experience as life-threatening.

Criterion A, as worded in DSM-III and DSMIII-R, has been considered deeply unsatisfactory in many ways by many experts on PTSD (Simpson, 1993): the reference to the "range of normal human experience" is obscure and was never based on any adequate scientific evidence.

Among the absurd suggestions in DSM was that clinicians should assess the stress based on what an "average" person "in a similar situation and with similar sociocultural values" would experience a highly fanciful exercise (Simpson, 1993) that ignores the patient's impression. Prevalence of a stressor has nothing to do with its potential to engender PTSD: rape, child abuse, and crime are far from outside the range of normal human experience. Where a patient meets every clinical criterion for the diagnosis, are we to reject it 
because a comfortable and privileged clinician decides that the experience was not rare enough or nasty enough?

Lillywhite \& Neal also complain that "PTSD is a condition which presents with a wide spectrum of severity". Surely there is not condition that cannot present with a wide spectrum of severity? Assessment of the severity of the condition is routine, but not inevitably part of the diagnosis. They urge the use of one particular scale out of several available, because it includes a severity scale. There is no compelling evidence favouring the CAPS-1 scale over other PTSD measures. Earlier reliable and valid scales were to some extent outdated by the changes introduced in DSM-III-R, but other current measures, such as the PTSD Symptom Scale (PSS; Foa et al, 1993), also include severity measures; scales which do not can be supplemented by assessment of severity. Psychometric instruments which essentially merely ask whether the criteria for DSM PTSD are present can be valid indicators of the presence of PTSD without much risk of being interesting or of adding much to our knowledge. The possible value of instruments using dichotomous and continuous scales, which they suggest, has long been recognised (e.g. Weisenberg et al, 1987).

Lillywhite \& Neal refer to finding "marked clinical improvement" due to simple assessment (a therapeutically trivial intervention). This is so much at variance with experience of PTSD as to raise doubts about the diagnosis and severity of the cases to which they refer, and if these findings, which they report devoid of detail, are sound, they should publish them as soon as possible, and in full detail.

They would have been more justified in challenging the evidence for the efficacy of the eyemovement desensitisation technique reported by Specor \& Huthwaite. No plausible rationale has been offered for this therapy, while sensational claims are made, and it is being widely marketed and taught before adequate convincing and scientifically sound studies have established its efficacy. Metter \& Michelson (1993), among others, have raised doubts about such excessive claims; and report a study in which the results of eye-movement desensitisation were not significantly different from those of control conditions, and "possibly less efficacious than having subjects stare at a dot on the wall."

EPSTEIN, R.S. (1993) Avoidant symptoms cloaking the diagnosis of PTSD in patients with severe accidental injury. Journal of Traumatic Stress, 6, 451-458.
Feinstein, A. \& Dolan, R. (1991) Predictors of post-traumatic stress disorder following physical trauma: an examination of the stressor criterion. Psychological Medicine, 21, 85-91.

FoA, E.B., Rigos, D.S., DANCU, C.V., et al (1993) Reliability and validity of a brief instrument for assessing post-traumatic stress disorder. Journal of Traumatic Stress, 6, 459-473.

MARCH, J.S. (1993) What constitutes a stressor? The "Criterion A" issue. In Posttraumatic Stress Disorder: DSM-IV \& Beyond (eds J.R.T. Davidson \& E.B. Foa), pp. 37-54. Washington: American Psychiatric Press.

Metter, J. \& Michelson, L.K. (1993) Theoretical, clinical, research and ethical constraints of the eye movement desensitization reprocessing technique. Journal of Traumatic Stress, 6, 413-415.

SimPSON, M.A. (1993) Traumatic stress and the bruising of the soul: The effects of torture and coercive interrogation. In The International Handbook of Traumatic Stress Syndromes (eds J.P. Wilson \& B. Raphael), pp. 667-684, New York: Plenum Press. Weisenderg, M., Solomon, Z., Schwarzwald, J., et al (1987) Assessing the severity of posttraumatic stress disorder: relation between dichotomous and continuous measures. Journal of Consulting and Clinical Psychology, 55, 432-434.

Michael A. Simpson

Centre for Psychosocial and Traumatic Stress

Pretoria Department of Psychiatry

Medical University of South Africa

\section{Diogenes syndrome by proxy}

SIR: There has been considerable interest in factitious disorders both in the lay press and among psychiatric nosologists in the preparation of DSM-IV. This interest has focused mainly on Munchausen syndrome and Munchausen syndrome by proxy. Diogenes syndrome, which refers to a state of marked self-neglect, domestic squalor and disorder, hoarding rubbish and social isolation, is well described since the term was first used by Clark et al (1975). However, a factitious disorder involving the classic features of Diogenes syndrome by proxy has not been described. We now report such a case.

Case report. An 81-year-old woman was referred by her general practitioner for in-patient assessment of mobility, having been bed-bound for two weeks following a fall. The fall resulted from poor sight due to senile macular degeneration. She also suffered from a chronic anxiety state, and she had complained of "low spirits".

The patient was moderately anxious, had faecal impaction and impaired visual acuity, but was independently mobile with the aid of a walking frame. She complained that during the two weeks of being bed-bound she had been neglected by her 48 -year-old daughter, who had lived with the patient for the previous year. The patient alleged having been deprived of regular food, clean bed linen and assistance with getting to the toilet, resulting in double incontinence. On further questioning, it became clear that the patient's immobility was compounded by physical obstruction of her pathway from her bed to the bathroom 